\title{
Estrutura, relações e antecedentes do uso de drogas em famílias de usuários de crack*
}

\author{
Structure, relationships and history of drug use in families of crack cocaine users
}

Estructura, relaciones y antecedentes del uso de drogas en familias de usuarios de crack

Maycon Rogério Seleghim ${ }^{1}$, Magda Lúcia Félix de Oliveira ${ }^{2}$

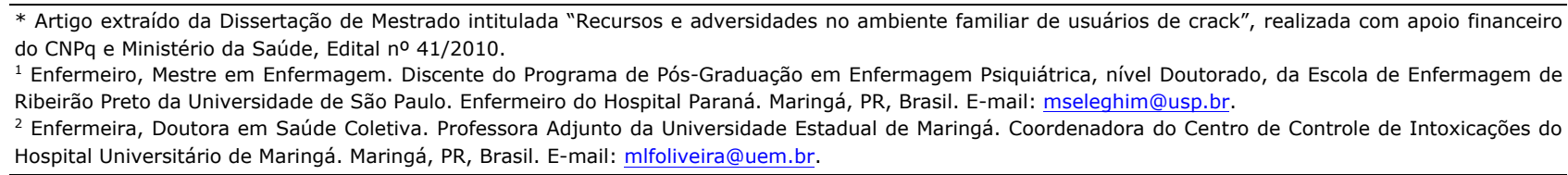

\section{RESUMO}

O objetivo foi analisar a estrutura, as relações e os antecedentes do uso de drogas em famílias de usuários de crack. Pesquisa transversal, com enfoque na Teoria Geral dos Sistemas, particularmente o uso do genograma. Participaram 15 familiares de usuários de crack acompanhados em um serviço especializado para o tratamento da dependência química do Paraná, no mês de maio de 2011. Utilizou-se entrevista semiestruturada com elaboração de genogramas, os quais foram agrupados por núcleos de similaridade. Os participantes foram, em sua maioria, mães com baixa escolaridade. Quanto à estrutura familiar, verificou-se maior número de pessoas na segunda geração, sendo esta constituída por famílias nucleares, e a primeira geração, por diferentes configurações. Os relacionamentos e os antecedentes familiares do uso de drogas acompanharam as diferenças geracionais encontradas na estrutura. Concluise que as características das famílias dos usuários de crack parecem acompanhar as mudanças ocorridas na estrutura social nas últimas décadas.

Descritores: Características Familiares; Relações Familiares; Cocaína Crack; Serviços Comunitários de Saúde Mental; Enfermagem de Família.

\section{ABSTRACT}

The objective of this study was to analyze the structure, relationships and history of drug use in families of crack cocaine users. It is a cross-sectional study, based on the General Systems Theory and on the genogram. The sample comprised 15 families of crack cocaine users cared for by a specialized drug dependency service in the state of Paraná, Brazil, throughout the month of May 2011. We conducted semi-structured interviews and created genograms, which were grouped according to similarities. For the most part, participants were mothers with low education levels. Regarding family structure, we found most people belonged to the second generation, constituting nuclear families, and to the first generation, with different family configurations. Relationships and family history of drug use followed the generational structural differences. We conclude that the characteristics of crack cocaine users' families seem to follow the changes in social structure observed over the last few decades.

Descriptors: Family Characteristics; Family Relations; Crack Cocaine; Community Mental Health Services; Family Nursing.

\section{RESUMEN}

Se objetivó analizar estructura, relaciones y antecedentes del uso de drogas en familias de usuarios de crack. Investigación transversal, con enfoque en la Teoría General de los Sistemas, utilizándose particularmente el genograma. Participaron 15 familiares de usuarios de crack seguidos por servicio especializado en tratamiento de dependencias químicas de Paraná, en mayo de 2011. Se usó entrevista semiestructurada con elaboración de genogramas, agrupados por núcleos de similitud. Los participantes fueron, mayoritariamente, madres con baja escolarización. Respecto a la estructura familiar, se verificó mayor número de personas en la segunda generación, constituyéndose ésta de familias nucleares, y la primera generación por diferentes configuraciones. Las relaciones y los antecedentes familiares del uso de drogas se correspondieron con las diferencias generacionales encontradas en la estructura. Se concluye en que las características de las familias de usuarios de crack parecen corresponderse con los cambios acontecidos en la estructura social en las últimas décadas.

Descriptores: Composición Familiar; Relaciones Familiares; Cocaína Crack; Servicios Comunitarios de Salud Mental; Enfermería de la Familia. 


\section{INTRODUÇÃo}

O crack é um tema atual e relevante para a área da saúde, bem como para outros setores da sociedade, devido à gravidade das consequências individuais, sociais e familiares associadas ao seu uso, e pela necessidade de ações de enfrentamento seja no âmbito do tratamento, da prevenção ou da redução de danos ${ }^{(1)}$.

Quanto à epidemiologia do uso, pesquisas realizadas nas ultimas décadas tem apontado para o aumento do número de usuários, desde os primeiros relatos de sua introdução no Brasil no final da década de $1980^{(1-2)}$. Comparando-se dois estudos de levantamento sobre o uso de drogas em grandes cidades, observa-se que em 2005 a proporção de indivíduos que consumiram crack alguma vez na vida era de 0,7\%, e em 2012 essa proporção passou para 2,2\%, o que corresponde a 2,8 milhões de consumidores da droga ${ }^{(3-4)}$.

Entre os fatores contextuais de risco para o uso de crack, as relações e os antecedentes familiares de uso de drogas são importantes aspectos que favorecem à aproximação inicial e à continuidade ao uso de crack e de outras substâncias psicoativas $(\mathrm{SPA})^{(5-6)}$. Como aponta estudo realizado com o objetivo de analisar a influência do ambiente familiar no consumo de crack em usuários, que encontrou que as famílias estudadas apresentaram vários elementos considerados desfavoráveis, os quais atuaram como elemento facilitador ao uso de crack, como a deficiência de suporte parental, a superproteção dos filhos, e a presença de cultura implícita do uso de drogas ${ }^{(6)}$.

No entanto, em relação à literatura internacional, observa-se carência de estudos nacionais sobre a interface das famílias com o uso de crack, incluindo a identificação de características familiares básicas, como por exemplo, a dinâmica e a composição familiar, além do histórico do uso de drogas dessa população(1,5-7).

Assim, considerando a família como uma unidade primordial no âmbito da construção, e desenvolvimento dos indivíduos que a compõem, transmitindo às gerações valores, regras, costumes, modelos e padrões de comportamentos, inclusive hábitos nocivos à saúde, torna-se necessário a investigação dos múltiplos aspectos dessa temática, na tentativa de contribuir para a formulação de políticas públicas específicas de prevenção ao uso do crack e de outras drogas com enfoque nos aspectos familiares ${ }^{(6,8)}$.

Diante disso, o objetivo do presente estudo é analisar a estrutura, as relações e os antecedentes do uso de drogas em famílias de usuários de crack.

\section{MÉTODOS}

Pesquisa transversal, que teve como aporte teórico a Teoria Geral dos Sistemas (TGS), particularmente o uso do genograma. De acordo com essa teoria, o comportamento de cada um dos membros familiares é interdependente do comportamento dos outros, e o que aconteceu em uma geração tende a acontecer nas demais $^{(9)}$.

O genograma é instrumento que permite retratar a estrutura familiar, os padrões de relacionamentos, manutenção de conflitos, bem como reunir dados de sua história. É, portanto, uma forma gráfica de apresentar informações que permite rápida apreensão de complexos padrões familiares e a reflexão de como questões clínicas podem estar ligadas à evolução de problemas e da própria família ao longo do tempo ${ }^{(10)}$.

Fizeram parte do estudo 15 familiares de usuários de crack acompanhados em um serviço especializado para o tratamento da dependência química, localizado na Região Noroeste do Estado do Paraná, no mês de maio de 2011. As famílias foram selecionadas indiretamente (amostra intencional), por critérios de usuários de crack em tratamento no referido serviço, ao considerar como critério de inclusão/exclusão: idade igual ou superior a 18 anos e presença de vínculo familiar, sendo estas informações obtidas com os profissionais do serviço.

Após essa identificação preliminar, os usuários foram abordados em uma sala privativa do serviço para informá-los sobre a realização do estudo e solicitar autorização para a abordagem familiar. As famílias foram convidadas a participar do estudo por meio de contato telefônico, onde era explicitado os motivos do contato e os objetivos do estudo. Assim, 10 familiares foram entrevistados no próprio serviço e cinco em seus domicílios.

Respeitando a autonomia das famílias, as mesmas elegeram entre seus membros, um informante familiar para participar da entrevista. O roteiro de entrevista semiestruturada foi constituído pelos seguintes itens: 1) dados pessoais do informante familiar - sexo, parentesco, idade, situação civil, filhos, escolaridade, religião e situação ocupacional; 2) características familiares - classificação econômica, religião, tipo de assistência à saúde, e atividades recreacionais; e 3) desenho do genograma - configuração, relacionamento e antecedentes familiares de uso de drogas de abuso.

$O$ instrumento de classificação econômica utilizado foi o Critério de Classificação Econômica Brasil, que divide as classes econômicas em: $A 1(R \$ 11.490,00)$, $A 2(R \$ 8.295,00), B 1(R \$ 4.754,00), B 2(R \$ 2.656,00)$, 
C1 $(R \$ 1.459,00), C 2(R \$ 962,00), D(R \$ 680,00)$, e $E$ $(R \$ 415,00)^{(11)}$. O modelo gráfico utilizado para construção dos genogramas foi adaptado de autores que trabalham com esta técnica de coleta de dados, sendo o desenho dos genogramas realizado manualmente pelo pesquisador, após explicação sobre a sua finalidade e a apresentação dos símbolos que poderiam ser utilizados (Figura 1$)^{(9)}$.

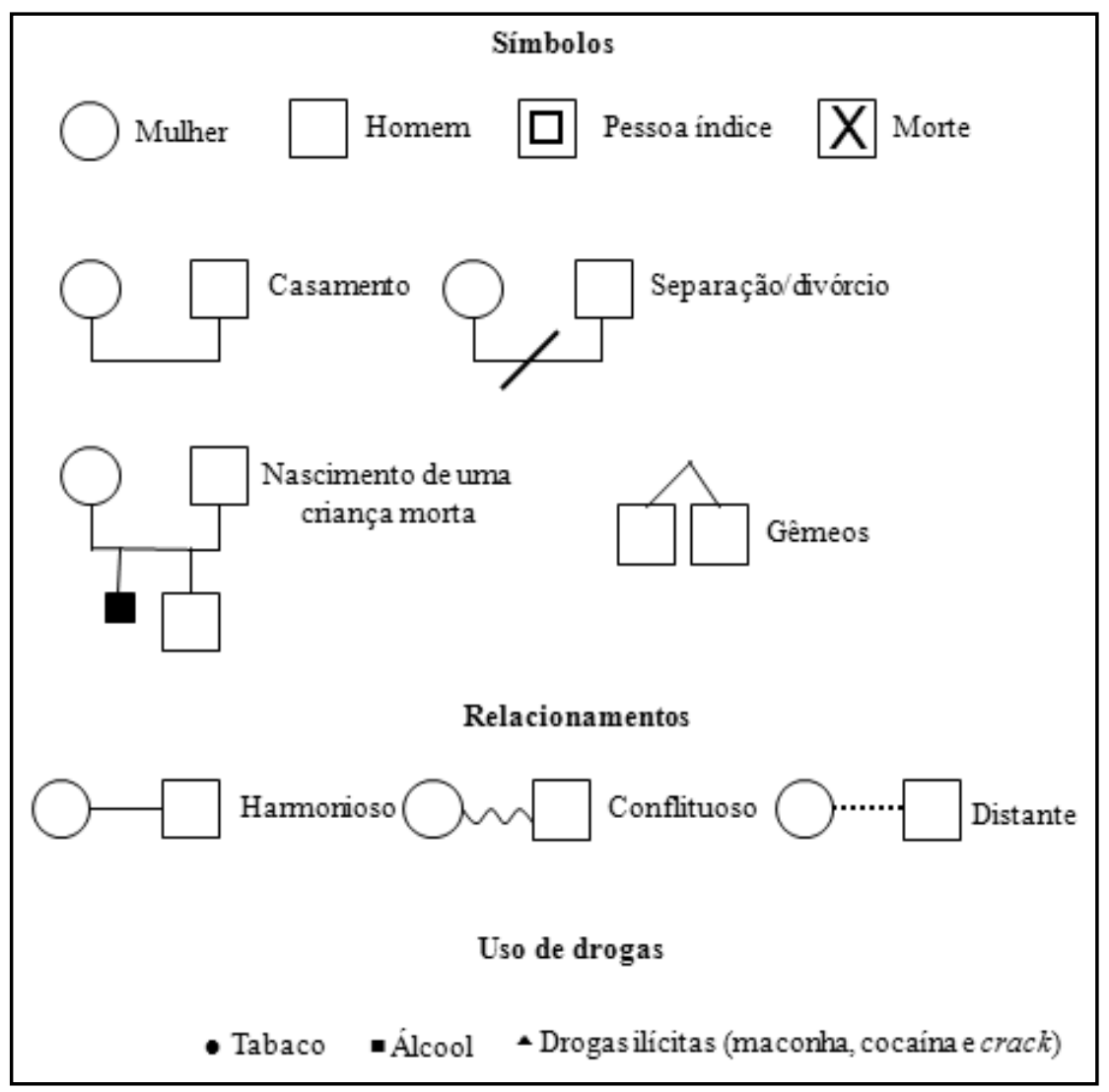

Figura 1: Formas gráficas utilizadas para a construção dos genogramas.

Inicialmente, procedeu-se à elaboração da geração familiar do sujeito índice ( $1^{a}$ geração) - pais e irmãos, e a elaboração da geração antecessora (2a geração) avós e tios. Em seguida, os familiares foram questionados sobre o uso de drogas lícitas e ilícitas pelos membros da família, e o tipo de relacionamento que eles possuíam com cada pessoa. Ao final de cada genograma, os entrevistados puderam completar ou alterar o desenho inicial.

Para caracterização do relacionamento familiar foram utilizados três tipos de representação(10): 1) relacionamento harmonioso: experiência emocional de união entre os membros familiares que nutrem sentimentos positivos e que possuem interesses, atitudes ou valores recíprocos. 2) relacionamento conflituoso: relações nas quais há constantes atritos que geram muita ansiedade e desavenças no meio familiar; e 3) relacionamento distante: caracteriza-se por pouco contato, principalmente de ordem emocional.

Cada entrevista, com duração aproximada de 60 minutos, foi realizada em ambiente privativo, gravada na sua totalidade e transcrita literalmente. Os dados pessoais dos informantes e das famílias foram tabulados e analisados por meio de estatística descritiva simples, e serão apresentados por meio de números absolutos.

Os genogramas foram incluídos no programa Power Point $^{\circledR}$, e para sua análise foi conduzido um processo semelhante ao da análise de conteúdo: todos os genogramas foram cuidadosamente observados, de modo a ser possível agrupá-los em núcleos de similaridade $^{(9)}$. Desse modo, os dados obtidos foram agrupados em três unidades de análise: a estrutura familiar dos usuários de crack; o relacionamento familiar na perspectiva da família, e os antecedentes familiares do uso de drogas de abuso.

Cada entrevista foi codificada por meio da letra ' $F$ ' seguida de algarismos arábicos conforme a sequência de realização das mesmas. A realização do estudo foi autorizada pela CT e aprovado pelo Comitê Permanente de Ética em Pesquisas com Seres Humanos da Universidade Estadual de Maringá (Parecer no 301/11). 


\section{RESULTADOS}

Os informantes familiares foram em sua maioria mães (nove sujeitos) e irmãos (três sujeitos). A idade dos participantes no estudo variou de 19 a 62 anos, sendo que a maioria era casada (11 sujeitos), possuía mais de um filho (12 sujeitos), tinha em média 7,9 anos estudados, era católica (11 sujeitos), e estava trabalhando no momento da entrevista (nove sujeitos).

Em relação às características familiares, a maioria das famílias pertencia à classe econômica $C$ (sete famílias) ou B (sete famílias), independente de suas subdivisões. Somente três famílias não eram adeptas a nenhuma religião, sendo que a maioria era católica. A utilização do Sistema Único de Saúde (SUS) foi a mais relatada pelas famílias (10 famílias), no entanto, algumas (cinco famílias) faziam a utilização do sistema privado. Dentre as atividades recreacionais realizadas em conjunto pelas famílias, o almoço familiar foi a de maior frequência (12 famílias).

\section{A estrutura familiar dos usuários de crack}

De um modo geral, a composição familiar das 15 famílias investigadas por meio da elaboração dos genogramas incluíram duas gerações com um total de 378 pessoas e uma média de 25,2 pessoas por família número mínimo de 13 e máximo de 39 pessoas.

Por outro lado, verificou-se um decréscimo de pessoas comparando a segunda (avós e tios) com a primeira geração familiar (pais e irmãos), visto que a segunda foi constituída por 245 familiares (média de 20,4 ) e a primeira por 133 pessoas (média de 8,8).

Essa diferença entre as gerações também pode ser evidenciada na configuração familiar, visto que a segunda geração foi constituída em sua totalidade por famílias nucleares, formada por um homem, uma mulher e seus filhos (biológicos ou adotivos), e a primeira geração apresentou novas e diversificadas configurações, pois nove apresentaram uma família nuclear; quatro monoparental, com uma estrutura formada de pais únicos devido principalmente ao divórcio, ao óbito e a "reprodução autônoma"; e apenas duas foram comunitárias, constituídas por pessoas com vínculos afetivos e familiares diversos.

\section{O relacionamento familiar na perspectiva do informante}

Considerando a tipologia de relacionamento que orientou a elaboração dos genogramas (relacionamentos harmoniosos, distantes e conflituosos), verificou-se certa relação entre a estrutura familiar e a ocorrência dos relacionamentos.

Isso pode ser percebido, pois apesar de todas as famílias apresentarem relacionamentos harmoniosos hegemonicamente em todas as gerações, verificou-se que os relacionamentos distantes (11 famílias) foram mais frequentes na segunda geração (nove famílias) e menor na primeira (duas famílias), e os relacionamentos conflituosos mais frequentes na primeira geração (oito famílias) (Figura 2).

\section{Os antecedentes familiares de uso de drogas}

o uso de drogas foi uma característica frequentemente encontrada nos genogramas analisados, visto que quase a totalidade das famílias (14) apresentou antecedentes de uso de drogas lícitas (seis famílias) e ilícitas (oito famílias) (Figura 3 ). A relação do uso de drogas e a geração familiar também corroboraram os resultados encontrados na estrutura e no relacionamento familiar, pois a maioria dos consumidores (80 pessoas identificadas nos 15 genogramas) encontrava-se na segunda geração.

No que se refere ao uso de drogas lícitas como o álcool e o tabaco, 91 pessoas, quase um quarto das pessoas "desenhadas" nos genogramas, foram identificadas como consumidores, com uma média de 6,0 pessoas por família, sendo a maioria (61) também pertencentes a segunda geração. Já o uso de drogas ilícitas, principalmente maconha, cocaína e crack, foi mais frequente na primeira geração (16 pessoas), com uma média de 1,1 por família. 


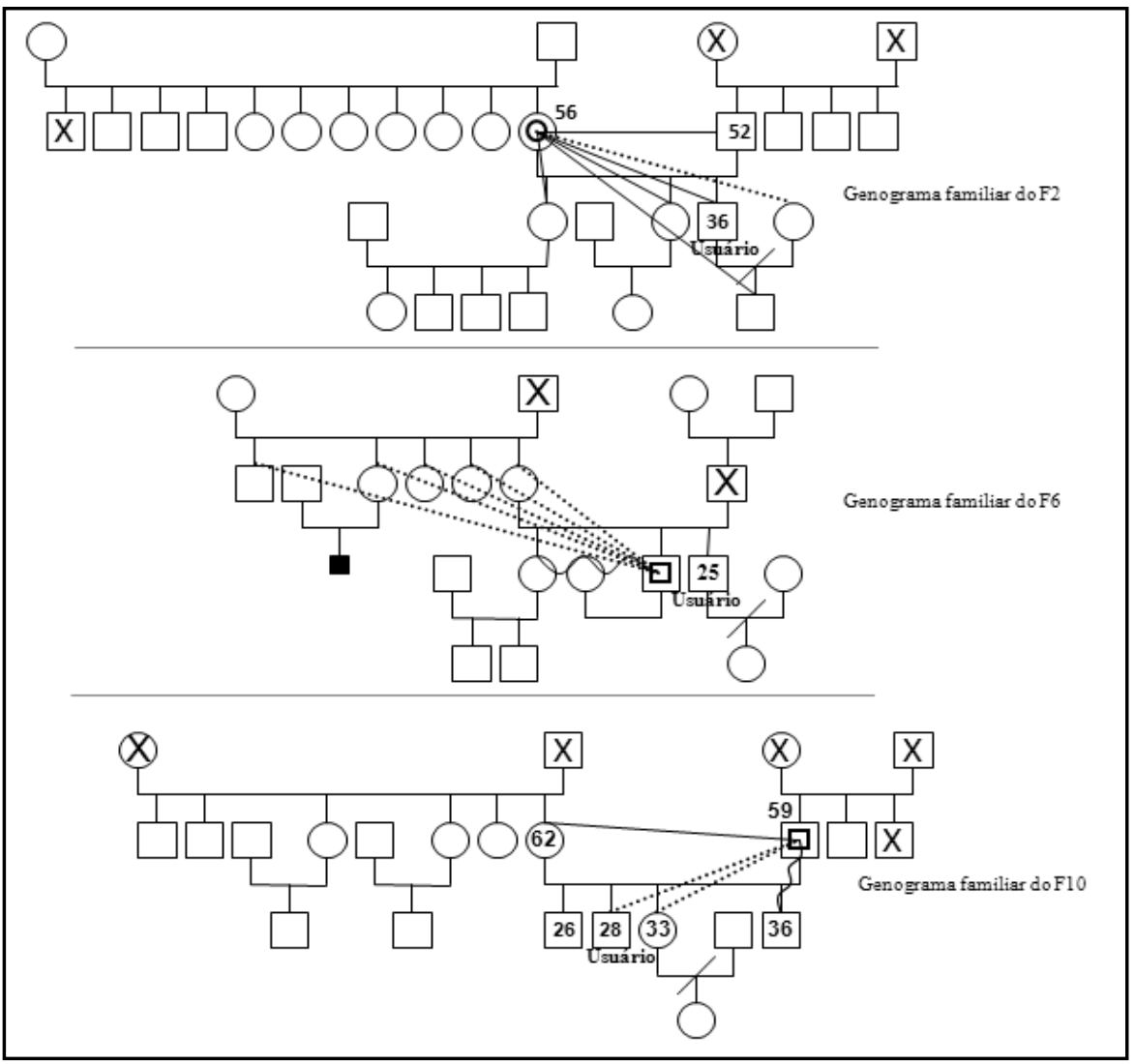

Figura 2: Genograma familiar dos informantes F2, F6, e F10.

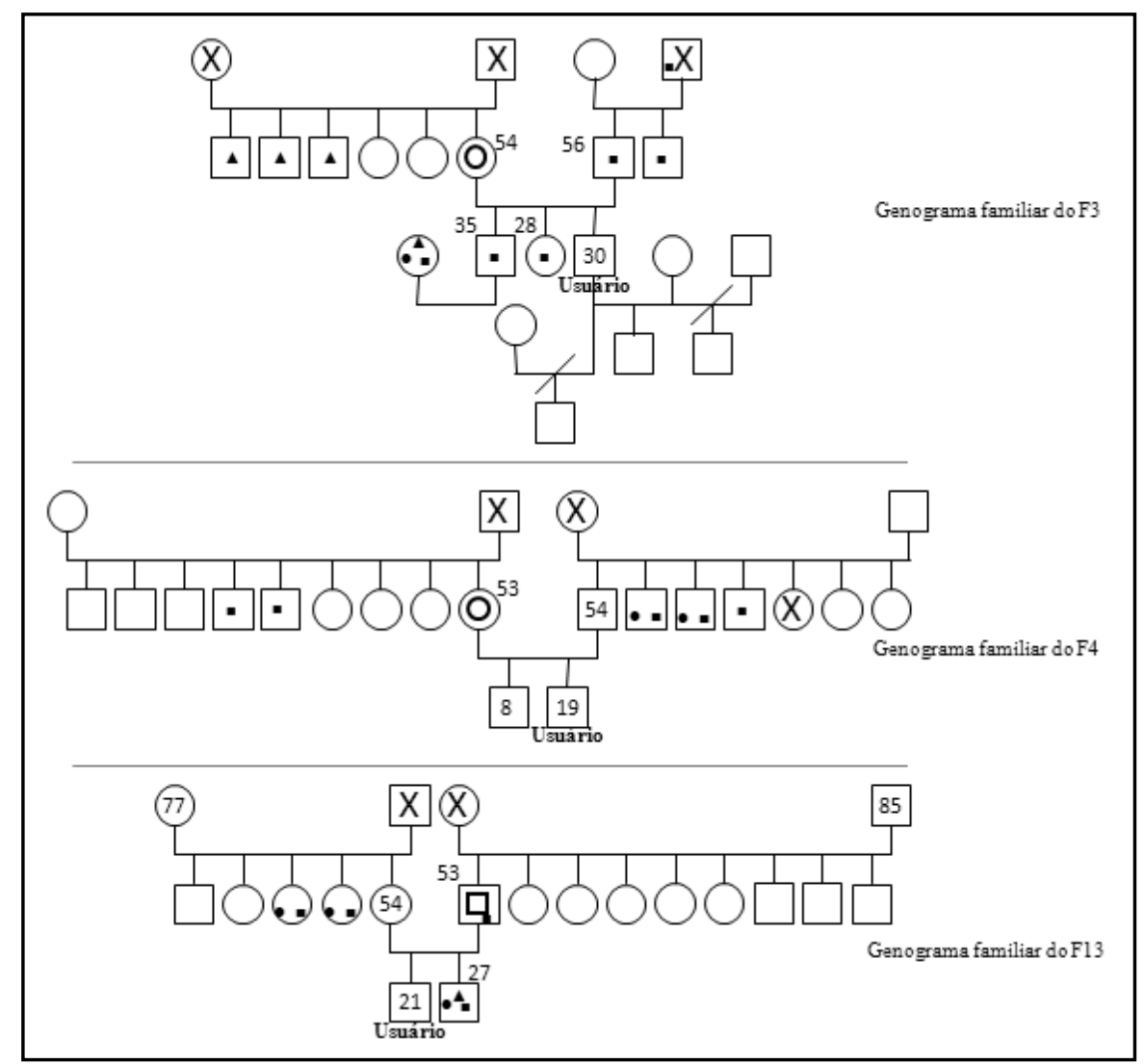

Figura 3: Genograma familiar dos informantes F3, F4, e F13.

\section{DISCUSSÃO}

Os informantes familiares foram em sua maioria mães, escolhidas pelo grupo familiar para participar da pesquisa. Essa maior ocorrência pode ser devido ao fato de que a mulher sempre ocupou um papel importante dentro das famílias, permitindo as trocas afetivas 
marcantes para o indivíduo e decisórias no modo de ser e agir consigo mesmo e com os outros, possuindo ainda importante papel no fenômeno do uso de drogas ${ }^{(12)}$.

Também, dados apontam que no período de 2000 a 2010, houve um crescimento expressivo das famílias com a pessoa responsável do sexo feminino, de 22,2 para $37,3 \%$. Os motivos para esse aumento podem ser creditados a uma mudança de valores culturais, relativo ao papel da mulher na sociedade brasileira. 0 ingresso maciço no mercado de trabalho e o aumento da escolaridade em nível superior combinado com a redução da fecundidade, são fatores que podem explicar esse reconhecimento da mulher como responsável pela família ${ }^{(13)}$.

Com relação às classes econômicas das famílias estudadas, verificou-se que quase a totalidade das famílias pertencia à classe $\mathrm{B}$ ou $\mathrm{C}$, independentemente de suas subdivisões, abrangendo uma renda de $\mathrm{R} \$ 962,00$ a $\mathrm{R} \$ 4.754,00$. Isso corrobora os dados da Pesquisa Nacional de Amostra por Domicílios (PNAD), realizada em 2012, que encontrou que 44,6\% dos domicílios brasileiros tem um rendimento mensal de até um salário mínimo ( $R \$ 622,00), 27,6 \%$ entre um e dois salários, e $22 \%$ mais que dois salários ${ }^{(14)}$.

A maioria das famílias utilizava o SUS como sistema de assistência à saúde de seus membros. Autores apontam que o SUS tem se deparado com o aumento do número de usuários de crack que procuram tratamento, exigindo uma rede estruturada de atenção com garantia de continuidade da assistência ${ }^{(5)}$. No entanto, apesar de a redefinição do modelo de atenção à Saúde Mental no país representar um avanço, no sentido de evitar que os doentes fossem excluídos da sociedade, o Brasil ainda não foi capaz de criar serviços substitutos adequados e em quantidade compatível com a demanda ${ }^{(5)}$.

Quanto a estrutura familiar, este estudo encontrou que a segunda geração dos usuários de crack era constituída por famílias numerosas, além de possuir uma configuração "tradicional"/nuclear. Paralelamente, verificou-se que a primeira geração apresentou outros tipos de configurações além da nuclear, indicando que a estrutura familiar dos usuários de crack parece ter acompanhado as transformações ocorridas na sociedade.

Isso remete ao fato de que até a algumas décadas atrás as famílias poderiam ser caracterizadas como um grupo de pessoas que interagiam em uma rede completa de parentesco, de pelo menos três gerações ${ }^{(15)}$. Atualmente, essa compreensão de famílias necessita ser ampliada para incluir novas configurações e vínculos familiares.
Essas transformações no interior das famílias podem ser entendidas, principalmente, pelas mudanças que ocorreram na rede social, sendo de natureza política, econômica, religiosa e jurídica. Entende-se, então, que é na confluência desses diversos aspectos presentes na teia social que se deve compreender os novos modos de ser família ${ }^{(16)}$.

A variedade de configurações assumidas pelas famílias exige a necessidade de compreendê-la não como entidades fixas, mas como formas variadas de manifestação, assim, "[...] não se pode falar de família, mas de famílias, para que se possa tentar contemplar a diversidade de relações que se convivem na sociedade $^{\prime(17)}$.

Os relacionamentos familiares acompanharam as diferenças geracionais na estrutura e na configuração familiar, visto que os relacionamentos distantes foram relatados com maior frequência na segunda geração. Essa "distância" relatada pelos informantes apareceu justificada pela pouca proximidade e/ou convivência com estes familiares, mas também pode ser explicada pelo elevado número de pessoas pertencentes a esta geração. Já os relacionamentos conflituosos foram mais frequentes na primeira geração, podendo ser devido à maior relação de proximidade entre os constituintes familiares.

Apesar da ocorrência de relacionamentos distantes entre os membros familiares, verificou-se a ocorrência de relacionamentos harmoniosos em todas as famílias e em todas as gerações, indicando que apesar da falta de convívio, das dificuldades familiares existentes, ou de outros motivos que justifiquem os relacionamentos distantes e conflituosos entre os membros, evidenciou relacionamentos de forma a preservar a integridade familiar.

Relações familiares saudáveis desde o nascimento da criança servem como fator de proteção para toda a vida. Os padrões saudáveis de relações familiares associam-se intrinsecamente a uma rede de apoio que possa ser ativada, em momentos críticos, fomentando o sentimento de pertença, a busca de soluções e atividades compartilhadas ${ }^{(18)}$.

O vínculo e a interação familiar saudável servem de base para o desenvolvimento pleno das potencialidades dos indivíduos. Estudos mostram que os padrões de relação familiar, a atitude e o comportamento dos pais e irmãos são modelos importantes para os adolescentes, inclusive em relação ao uso de drogas. A interação familiar gratificante é um forte fator protetor, mesmo no caso onde os pais são usuários de drogas, quando esses 
são capazes de prover um contexto amoroso, afetuoso e de cuidado ${ }^{(18)}$.

Em relação aos antecedentes familiares do uso de drogas, chamou atenção que quase a totalidade das famílias estudadas tinha história de uso de substâncias lícitas e ilícitas, corroborando a ideia de que a presença de antecedentes familiares de uso de SPA podem ser importantes fatores de risco para a fase de experimentação e continuidade ao uso de drogas ${ }^{(19-20)}$.

Outro aspecto importante encontrado foi que a maioria dos relatos de uso de drogas lícitas estavam presentes na segunda geração e do uso de drogas ilícitas, como a maconha, cocaína e crack, estavam presentes na primeira geração.

Dentre as possíveis causas para este fato, além do número elevado de pessoas na segunda geração, que poderia ter uma relação estritamente numérica, tem-se observado nas últimas décadas um maior controle da comercialização e marketing das bebidas alcoólicas e do tabaco, o que poderia justificar o menor número de pessoas usuárias dessas substâncias na primeira geração.

Do mesmo modo, o maior número de pessoas usuárias de drogas ilícitas, como o crack, na primeira geração, poderia ser explicado pela recente introdução dessa droga em território brasileiro, mas também pela escolha intencional da amostra (famílias de usuários de crack), exigindo assim estudos mais abrangentes sobre a relação intergeracional do $\mathrm{crack}^{(20)}$.

\section{CONCLUSÃO}

Os resultados deste trabalho sugerem que as características das famílias dos usuários de crack,

\section{REFERÊNCIAS}

1. Raupp L, Adorno RC. Crack usage circuits in the downtown area of the city of São Paulo (SP, Brazil). Cienc. saude colet. 2011;16(5):2613-22.

2. Volcov K, Vasconcellos MP . "Crack, é possível vencer" ou é preciso compreender: observações a partir de campanhas publicitárias do governo federal. Sau. \& Transf. Soc. 2013; 4(2):99-105.

3. Carlini EA et al. II levantamento domiciliar sobre o uso de drogas psicotrópicas no Brasil: estudo envolvendo as 108 maiores cidades do País - 2005. Brasília: Secretaria Nacional Antidrogas, 2006.

4. Abdalla RR, Madruga CS, Ribeiro M, Pinsky I, Caetano R, Laranjeira R. Prevalence of cocaine use in Brazil: data from the II Brazilian National Alcohol and Drugs Survey (BNADS). Addict Behav. 2014 Jan;39(1):297-301.

5. Seleghim MR, Marangoni SR, Marcon SS, Oliveira ML. Vínculo familiar de usuários de crack atendidos em uma unidade de emergência psiquiátrica. Rev Latino-am Enferm [Internet]. 2011 [citado 2012 Jan. 15]; 19(5): [cerca de 8 telas]. Disponível em: http://www.scielo.br/pdf/rlae/v19n5/pt_14.pdf. incluindo a composição e a dinâmica familiar, parecem ter acompanhado as transformações sociais das ultimas décadas, sendo, portanto, passível de outros tipos de arranjos na atualidade.

A presença mais marcante da mulher como fonte de renda e sobrevivência familiar, a família pouco numerosa, além de outros tipos de configurações familiares, parece delimitar os novos modos de viver em família na modernidade, se vinculando também a novos padrões de relacionamentos familiares. Assim, este estudo impacta para a produção do conhecimento e/ou para a prática na área das drogas, o fato de que as funções básicas desempenhadas pela instituição familiar no decorrer do processo de desenvolvimento psicológico dos indivíduos que fazem uso de crack, apresentam especificidades que devem ser levadas em consideração.

Observa-se, portanto, a necessidade da elaboração de políticas públicas específicas de prevenção ao uso de drogas no ambiente familiar, bem como programas de orientação familiar sobre os papéis familiares, que considerem, além de outros aspectos, as diversidades de configurações expressadas pelas famílias.

Sugere-se, a realização de estudos em serviços especializados para tratamento da dependência química por permitirem maior proximidade com o sujeito de pesquisa para o estabelecimento de vínculo, dando maior fidedignidade aos dados obtidos. Ainda, as limitações dos resultados deste estudo são inerentes ao método. A pesquisa qualitativa, utilizando-se de amostra intencional, limita os achados à população investigada, não permitindo generalização à população global ou inferências a outras populações.

6. Seleghim MR, Oliveira MLF. Influência do ambiente familiar no consumo de crack em usuários. Acta paul. enferm. 2013; 26(3): 263-268.

7. Sanchez ZVDM, Nappo SA. From the first drug to crack: the sequence of drugs taken in a group of users in the city of São Paulo. Subst Use Misuse. 2007;42(1):177-88.

8. Moreno RS, Ventura RN, Bretãs JRS. Ambiente familiar e consumo de álcool e tabaco entre adolescentes. Rev. paul. pediatr. 2009;27(4):354-360.

9. Mcgoldrick M, Gerson R, Shellenberger S. Genograms assessment and intervention. 3. ed. New York: WWNorton \& Company; 2008. 373 p.

10. Wendt NC, Crepaldi MA. A Utilização do genograma como instrumento de coleta de dados na pesquisa qualitativa. Psicol. reflex. crit. $2008 ; 21(2): 302-310$.

11. Associação Brasileira de Empresas de Pesquisa. Critério de classificação econômica Brasil. 2010. [acesso em 20 jul 2010]. Disponível em:

http://www.abep.org/novo/Utils/FileGenerate.ashx?id=197. 12. Oliveira EB, Bittencourt LP, Carmo AC. A importância da família na prevenção do uso de drogas entre crianças e 
adolescentes: papel materno. SMAD, Rev. eletrônica saúde mental alcool drog. 2008;4(2):1-16.

13. Instituto Brasileiro de Geografia e Estatística. Resultados Preliminares do Universo do Censo Demográfico 2010. Disponível em:

http://www.ibge.gov.br/home/estatistica/populacao/censo2010/r esultados_preliminares/preliminar_tab_uf_zip.shtm Acessado em 29 de abril de 2014.

14. Instituto Brasileiro de Geografia e Estatística. Pesquisa

Nacional por Amostra de Domicílios. Síntese de indicadores 2012. Disponível em:

ftp://ftp.ibge.gov.br/Trabalho_e_Rendimento/Pesquisa_Nacional _por_Amostra_de_Domicilios_anual/2012/Sintese_Indicadores/si ntese_pnad2012.pdf. Acessado em 29 de abril de 2014.

15. Horta RL, Horta BL, Pinheiro RT. Drogas: famílias que protegem e que expõem adolescentes ao risco. J. bras. psiquiatr. 2006;55(4):268-272.

16. Shaurich D. Compreensões de acadêmicos de enfermagem sobre família. Esc. Anna Nery. 2009;3(2):415-420.

17. Gomes MA, Pereira MLD. Família em situação de vulnerabilidade social: uma questão de políticas públicas. Cienc. saude colet. $2005 ; 10(20): 357-363$.

18. Bernardy CCF, Oliveira MLF, Bellini LM. Jovens infratores e a convivência com drogas no ambiente familiar. Rev Rene. 2011; 12(3):589-96.

19. Rudolph AE, Rudolpha AE, Jonesa KC, Latkinb C, Crawforda ND, Fuller CM. The association between parental risk behaviors during childhood and giving high risk networks in adulthood. Drug Alcohol Depend. 2011;118(2):437-443.

20. Fergusson DM, Boden JM, Horwood LJ. The developmental antecedents of illicit drug use: evidence from a 25-year longitudinal study. Drug Alcohol Depend. 2008;96(1):65-177.

Artigo recebido em 29/12/2012.

Aprovado para publicação em 10/04/2014.

Artigo publicado em 30/09/2014. 